\title{
Thermogel Delivers Oxaliplatin and Alendronate in situ for Synergistic Osteosarcoma Therapy
}

\author{
Yifu Sun ${ }^{1}, \mathrm{Ke} \mathrm{Li}^{2}$, Chen $\mathrm{Li}^{1 *}$, Ying Zhang ${ }^{3 *}$ and Duoyi Zhao ${ }^{2 *}$ \\ ${ }^{1}$ Department of Orthopedics, The Second Hospital of Jilin University, Changchun, China, ${ }^{2}$ Department of Orthopedics, \\ The Fourth Affiliated Hospital of China Medical University, Shenyang, China, ${ }^{3}$ Department of Orthopedics, Zhongshan \\ Hospital Affiliated to Xiamen University, Xiamen, China
}

OPEN ACCESS

Edited by:

Shixian $L V$

University of Washington,

United States

Reviewed by:

Hai Xiong,

Shenzhen University, China

Jingxiao Chen,

Jiangnan University, China

${ }^{*}$ Correspondence:

Chen L

chenliortho@jlu.edu.cn

Ying Zhang

xmzhangying@163.com

Duoyi Zhao

zhaoduoyidr@163.com

Specialty section:

This article was submitted to

Biomaterials,

a section of the journal Frontiers in Bioengineering and

Biotechnology

Received: 18 June 2020

Accepted: 21 August 2020

Published: 15 September 2020

Citation:

Sun Y, Li K, Li C, Zhang Y and Zhao D (2020) Thermogel Delivers Oxaliplatin and Alendronate in situ

for Synergistic Osteosarcoma

Therapy.

Front. Bioeng. Biotechnol. 8:573962

doi: 10.3389/fbioe.2020.573962
The therapeutic effect of osteosarcoma (OS) has not made extraordinary progress in the past few decades. Oxaliplatin (OXA) is a widely used clinical anti-tumor drug. Recent studies have shown that OXA can trigger anti-tumor immunity by inducing immunogenic death (ICD). Alendronate (ALN) has been used to threaten the skeletal system tumors because of the unique bone affinity and the ability to inhibit bone destruction. In this study, we co-loaded OXA and ALN on mPEG45-PLV19 thermo-sensitive hydrogel to perform in situ treatment on the mouse OS model. Slowly released OXA can induce immunogenic death of tumor cells. At the same time, thermo-sensitive hydrogels can induce the accumulation of cytotoxic T lymphocytes. Besides, ALN could synergistically diminish tumors and prevent bone destruction. This system could synergistically inhibit the progression of OS and lung metastasis and has no toxicity to various organs throughout the body.

Keywords: thermo-sensitive hydrogel, oxaliplatin, alendronate, immune activation, osteosarcoma therapy

\section{INTRODUCTION}

Osteosarcoma (OS), as the highest incidence of malignant bone tumor, has been harmful to human health (Wang et al., 2020). OS often occurs to adolescents (Tinkle et al., 2019), especially to boys. At present, the standard treatment of OS includes neoadjuvant chemotherapy, surgical resection, and post-operative chemotherapy (Zhang et al., 2020). Despite the deepening of research in this field, there has been no significant improvement in patient survival in recent decades (Harrison et al., 2018). Lung metastasis is an essential factor of the poor prognosis of OS (Huang X. et al., 2019).

In recent years, immunotherapy has provided new directions for the treatment of cancer. Researchers have found that some current chemotherapy drugs can induce immunogenic cell death (ICD) of tumor cells, and the mechanism is related to the induction to apoptosis, including the exposure of calreticulin (CRT) on the cell membrane (Sukkurwala et al., 2014). ICD would release tumor antigens, thereby triggering tumor immune response (Menger et al., 2012; Martins et al., 2014). Platinum drugs are the first-line chemotherapy drugs for the treatment of OS (Cheng et al., 2020). Oxaliplatin (OXA) is the third-generation platinum anti-tumor drug that 
target DNA as the site of action. Platinum atoms form a cross-link with DNA to antagonize its replication and transcription. Researchers have found that OXA could induce anti-tumor immunity, which produces anti-tumor immune effects by initiating ICD, acting on cellular STAT protein signaling pathway, and regulating the immunosuppression tumor microenvironment (Ghiringhelli et al., 2009; Hato et al., 2014). There is evidence that OS may be sensitive to immunotherapy. The percentage of infiltrating $\mathrm{CD}^{+} \mathrm{T}$ lymphocytes in OS was higher than that in other sarcoma subtypes (van Erp et al., 2017), and the degree of infiltration was positively correlated with survival rate of patients (Gomez-Brouchet et al., 2017). OS has a high level of genomic instability and expresses programmed cell death protein-1 ligand (PD-L1) (Koirala et al., 2016), indicating potential sensitivity to inhibitors of PD-1/PD-L1 pathway (Kansara et al., 2014; Mouw et al., 2017).

Bisphosphonate is a successfully bone resorption inhibitor, which has a strong affinity with hydroxyapatite in bone. It could inhibits the activity of osteoclasts, thus inhibits the bone destruction caused by OS (Blanco et al., 2009). Alendronate (ALN) is one of the most commonly used bisphosphates in the clinic (Uihlein and Leder, 2012). It is mainly used for the treatment of osteoporosis caused by menopause, glucocorticoid disorders, and parathyroid disease (Wang et al., 2017, 2018; Jiang et al., 2020). ALN has achieved satisfactory therapeutic effects in the above diseases.

Thermo-sensitive hydrogel is widely used in biological research (Wei et al., 2017; Liu et al., 2018; Wang C. et al., 2019; Wang Y. et al., 2019; Zhang et al., 2019). It has injectability, suitable gel-forming ability, excellent biocompatibility, degradability, and excellent drug loading capacity (Huang H. et al., 2019). Hydrogel delivery drugs have been extensively studied (Li et al., 2018; Zhang et al., 2018; Ning et al., 2019; Qiu et al., 2020; Zheng et al., 2020). Studies have confirmed that hydrogels are able to serve as an immune adjuvant to recruit immune cells after implantation (Wang Y. et al., 2019).

In this study, polyvaline (PLV) thermo-sensitive hydrogel loaded with OXA and ALN was used for the treatment of OS. We aim to induce the ICD of OS cells through the release of OXA in the tumor site, thereby promoting the tumor immune response. At the same time, PLV hydrogel can recruit immune cells to synergistically enhance the immunotherapeutic effect. In addition, ALN could synergistically treat tumors and protect bone tissue against the damage caused by tumor invasion.

\section{MATERIALS AND METHODS}

\section{Cells and Animals}

The mouse OS cell line K7M2 is routinely cultured in DMEM medium (Invitrogen) containing 10\% neonatal bovine serum (Beijing Solabe Technology Co., Ltd.), and the cells are collected until they grow to about $80 \%$. SPF grade BALB/c female mice (4 weeks) were purchased from Beijing Weitong Lihua Experimental Animal Technology Co., Ltd. for use in vivo antitumor experiments of PLV hydrogel. All mice were kept in a clean and pathogen-free environment, the temperature was maintained at $20-22^{\circ} \mathrm{C}$, and the light-dark cycle was $12 \mathrm{~h}$. All mice were used and handled according to the protocol approved by the Jilin University Animal Protection and Use Committee.

\section{Thermo-Sensitive Hydrogel Loaded With Drugs}

First, the terminally aminated polyethylene glycol monomethyl ether is prepared by the hydroxyl amination reaction of polyethylene glycol monomethyl ether. Take $30 \mathrm{~g}$ of $\mathrm{mPEG}$, dissolve it in $300 \mathrm{~mL}$ of dichloromethane, add $8.4 \mathrm{~g}$ of potassium hydroxide, and $14.17 \mathrm{~g}$ of p-toluenesulfonyl chloride, and stir them at room temperature for 7 days. After the completion of the reaction, the dichloromethane solution was collected in a separatory funnel and washed with saturated sodium chloride aqueous solution six times, each about $30 \mathrm{~mL}$. Collect the lower methylene chloride layer, add anhydrous magnesium sulfate, and dry overnight. The dried dichloromethane solution was suction filtered to remove magnesium sulfate. The resulting solution was concentrated to $100 \mathrm{~mL}$ by rotary evaporation, and then settled with 10 times volume of ice ether for three times. The intermediate product was collected by filtration through a Buchner funnel and then drained by a cold trap. Weigh the mass of the intermediate product, add the equal mass of $\mathrm{NH}_{4} \mathrm{Cl}$, and add 30 times the volume of ammonia to dissolve it, and stir for 7 days. Collect the solution in a separatory funnel, add sodium chloride until the ammonia solution is saturated, add dichloromethane for extraction, add about $60.0 \mathrm{~mL}$ each time, extract five times, collect the lower liquid, add anhydrous magnesium sulfate and dry overnight. Filtered with a sand core funnel, the product was concentrated to about $100.0 \mathrm{~mL}, 10$ times the volume of ice ether was settled three times, and the terminally aminated $\mathrm{mPEG}_{2000}-\mathrm{NH}_{2}$ was obtained after suction.

After that, L-Val NCA was prepared by triphosgene and L-valine in the tetrahydrofuran solution. Add $400 \mathrm{~mL}$ of tetrahydrofuran to a dried three-necked flask, then add $15 \mathrm{~g}$ of L-valine and $9 \mathrm{~g}$ of triphosgene, slowly ventilate nitrogen and place the device in a $60^{\circ} \mathrm{C}$ oil bath and stir for about $1 \mathrm{~h}$. After the solution is clear, add $15 \mathrm{~g}$ of L-valine and $9 \mathrm{~g}$ of triphosgene again, and continue the reaction. After about $2 \mathrm{~h}$, increase the nitrogen flow rate, blow the solution to about $100 \mathrm{~mL}$, and settle the reaction solution with a 10 -fold volume of $n$-hexane solution at a temperature of $-20^{\circ} \mathrm{C}$. After the sedimentation is complete, filter the resulting product with a funnel, collect the upper filter residue, and dissolve it with $200 \mathrm{~mL}$ of ethyl acetate at a temperature of $-20^{\circ} \mathrm{C}$. Collect the ethyl acetate solution in a separatory funnel, wash the solution with a saturated sodium bicarbonate solution at $4^{\circ} \mathrm{C}, 30 \mathrm{~mL}$ each time, wash six times, and collect the supernatant. Then wash the solution with $4^{\circ} \mathrm{C}$ water, $30.0 \mathrm{~mL}$ each wash six times, and collect the upper layer. Collect the washed ethyl acetate solution into an Erlenmeyer flask, add an appropriate amount of anhydrous magnesium sulfate, and place it in a refrigerator at $-20^{\circ} \mathrm{C}$ for about $4 \mathrm{~h}$.

The crude L-Val NCA was recrystallized. Filter the dried ethyl acetate solution with a sand core funnel into the dried bottle. After collecting the solution, connect the cold trap to drain the ethyl acetate to obtain crude L-Val NCA. The drained product 
A

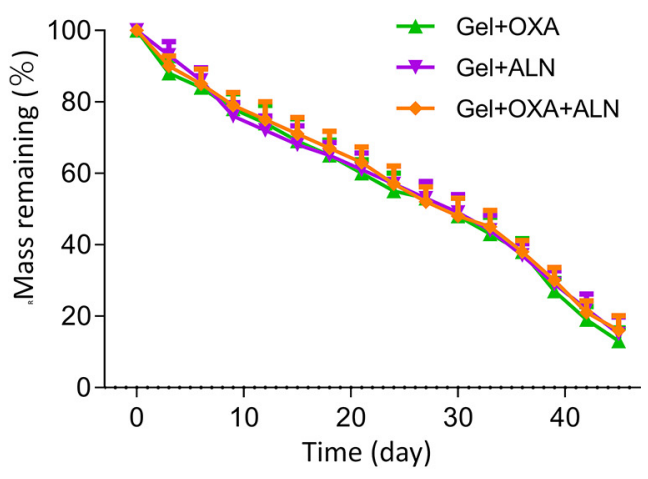

C

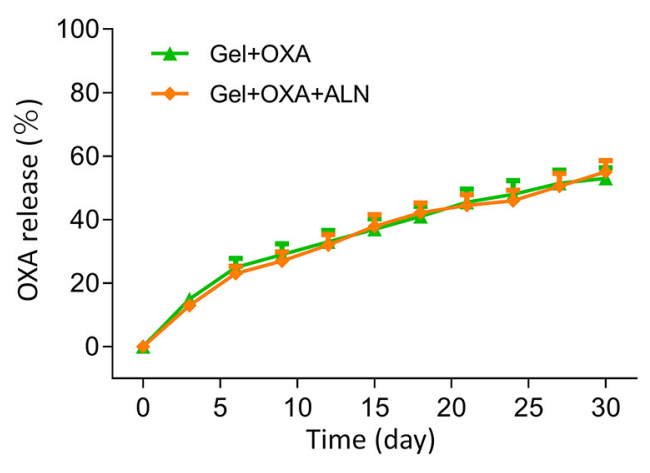

B

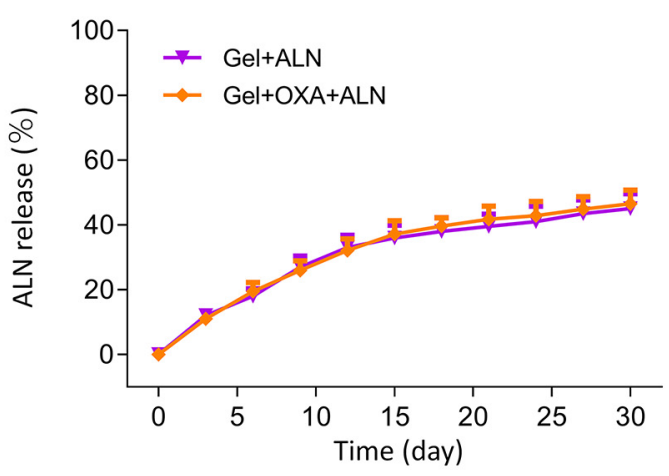

D

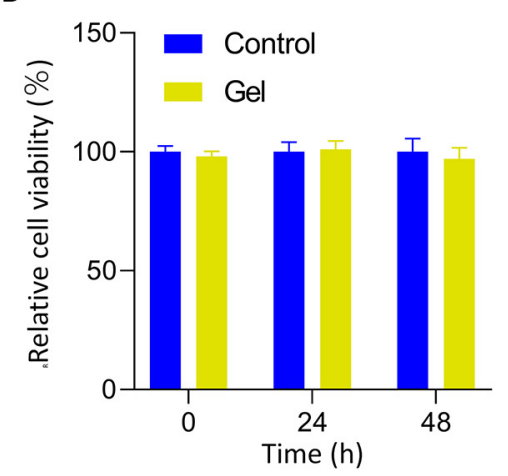

FIGURE 1 | (A) Mass remaining of each group Gel in vitro. Cumulative release curves of ALN (B) and OXA (C) from each group in vitro. (D) MTT assay was used to measure the effects of the Gel on viabilities of K7M2 cells at 0, 24, $48 \mathrm{~h}$ after dispose, the data is expressed as mean $\pm \operatorname{SD}(n=3)$.

was dissolved with $30 \mathrm{~mL}$ of tetrahydrofuran at $40^{\circ} \mathrm{C}$, and then $10 \mathrm{~mL}$ of $\mathrm{n}$-hexane solution was added until precipitation precipitated, and then dissolved at $60^{\circ} \mathrm{C}$. Subsequently, the recrystallized L-Val NCA solution was placed in a refrigerator at $-20^{\circ} \mathrm{C}$ overnight. The recrystallized L-Val NCA upper layer solution is drawn out and then drained by a cold trap to obtain refined L-Val NCA.

Finally, $\mathrm{mPEG}_{45}-\mathrm{NH} 2$ was used as a macroinitiator to initiate the ring-opening polymerization of L-valine NCA to obtain $\mathrm{mPEG}_{45}-\mathrm{PLV}_{19}$. Dissolve $2.86 \mathrm{~g}$ of L-Val NCA in $100 \mathrm{~mL}$ of DMF. Reacted at room temperature for 3 days, and then settled with $1000 \mathrm{~mL}$ of ice ether. The obtained product was dissolved in $40 \mathrm{~mL}$ of DMF and placed in a dialysis bag with a molecular weight cut-off of $5000 \mathrm{Da}$ for dialysis for 3 days. After freezedrying, the final product $\mathrm{mPEG}_{45}-\mathrm{PLV}_{19}$ was obtained.

$\mathrm{mPEG}_{45}-\mathrm{PLV}_{19}$ (5.0 wt\%) was stirred slowly and thoroughly at $4^{\circ} \mathrm{C}$ overnight, and OXA $(5.0 \mathrm{mg} / \mathrm{mL})$ (Feather et al., 2018) and ALN (10.0 mg/mL) (Shan et al., 2020) were added to the polymer solution and mixed thoroughly $\left(4^{\circ} \mathrm{C}\right)$ to keep injectable. The sol state is used for tumor suppression experiments.

\section{Degradation and Drug Release in vitro}

To simulate the degradation of the gel in the body, we chose a buffer solution prepared from PBS, elastase $(0.2 \mathrm{~g} / \mathrm{L})$, calcium chloride $(\mathrm{CaCl} 2,10.0 \mathrm{mmol} / \mathrm{L})$, and sodium azide $\left(\mathrm{NaN}_{3}\right.$,
$0.2 \mathrm{wt} \%$ ) as the degradation medium. Add $3 \mathrm{~mL}$ of buffer solution to the top of the gel. Change the buffer solution every 3 days and accurately weigh the remaining gel to measure the biodegradation rate.

Place the $\mathrm{mPEG}_{45}-\mathrm{PLV}_{19}(5.0 \mathrm{wt} \%)$ gel in a glass bottle and cover the gel with $3 \mathrm{~mL}$ PBS. Place the glass bottle in a constant temperature shaker at $37^{\circ} \mathrm{C}$. The PBS solution was changed every 3 days. Detect the release of OXA and ALN by elemental analysis (ICP).

\section{MTT Assay}

Transfer $100 \mu \mathrm{L}$ of K7M2 in the medium to a 96-well plate (5000 cells/well). After culturing for $24 \mathrm{~h}$, add $100 \mu \mathrm{L}$ of medium or $\mathrm{mPEG}_{45}-\mathrm{PLV}_{19}$ (5.0 wt\%) Gel. After treatment for 0, 24, and $48 \mathrm{~h}$, add $20 \mu \mathrm{L}$ of tetramethylazolium salt indicator $(5 \mathrm{mg} / \mathrm{mL}$ in PBS, $\mathrm{pH}$ 7.4) to each well, and incubate for another $4 \mathrm{~h}$ in the dark at $37^{\circ} \mathrm{C}$. Measure the absorbance of the solution at $490 \mathrm{~nm}$ on a microplate reader. Determine the relative survival rate of cells by comparing the absorbance of different groups. The data are expressed as mean $\pm \mathrm{SD}$.

\section{Establishment of Osteosarcoma Model and Animal Grouping}

K7M2 cells in the logarithmic growth phase were digested with trypsin, washed three times in pre-chilled PBS, and suspended 
in cold PBS, ready for the preparation of OS in situ model. Anesthetize the mouse and remove the right lower limb hairs to fully expose the front surface of the right tibia and knee joint. Flex the mouse's knee at a $90^{\circ}$ position, using a $1 \mathrm{~mL}$ syringe needle, slowly rotate to vertically penetrate the cortex of the right tibia, and insert the needle along the long axis of the tibia until it is inserted into the metaphysis of the tibia about $3.5 \mathrm{~mm}$. Sampler, inject $25.0 \mu \mathrm{L}$ of cell suspension $\left(2 \times 10^{6} \mathrm{~K} 7 \mathrm{M} 2\right.$ cells $)$ into the medullary cavity of the tibia. After the injection is completed, a certain pressure is applied to the injection site to prevent excessive pressure from causing the cells to ooze out. Monitor the tumor growth of the right leg of the mouse, and calculate and record the tumor region volume (V) using Eq. 1 (Li et al., 2020):

$$
V\left(\mathrm{~mm}^{3}\right)=\frac{4 \pi}{3} \times \frac{L}{2} \times\left(\frac{A P}{2}\right)^{2}
$$

$A P$ is the maximum length of the lateral diameter of the tumor measured along the horizontal axis; $L$ is the maximum longitudinal length of the tumor measured along the long axis of the tibia.

When the tumor region volume reached $500 \mathrm{~mm}^{3}$, OS model mice were grouped. The mice were randomly divided into five groups, namely control group, OXA + ALN group, Gel + OXA group, Gel + ALN group, and Gel + OXA + ALN group, four mice in each group. The doses of OXA and ALN are 5 and $10 \mathrm{mg} / \mathrm{mL}$, respectively, and $100 \mu \mathrm{L}$ of $5.0 \mathrm{wt} \%$ PLV thermosensitive sol (at $4^{\circ} \mathrm{C}$ ) evenly mixed with OXA or (and) ALN was injected slowly into the orthotopic OS tumor of mice Instead of the injection of $\mathrm{mPEG}_{45}-\mathrm{PLV}_{19}$ gel in the same amount of PBS, the control group was injected with the same amount of PBS. When the experiment ended (16 days), the mice were euthanized. All mice were processed according to the protocol approved by the Animal Protection and Use Committee of Jilin University.

\section{Data Collection of Evaluation Indexes for in situ Inhibition of Osteosarcoma}

From the 0th day of administration, the body weight and tumor volume of mice were recorded at the same time every day, and the tumor inhibition curve, as well as tumor inhibition rate curve, were drawn. The tumor volume is calculated according to Eq. 1. On the 16th day of the experiment, the mice were sacrificed, and the hind limbs of the tumor were photographed and weighed, and then micro-CT scans were taken.

\section{Micro-CT}

Scan the right calf specimens of tumor-bearing mice with microCT (micro-CT; Bruker, Skycan1172, Germany) and use computer software (Brook, Germany) for three-dimensional reconstruction to observe the bone destruction and further locate the tumor center Projection of the site on the anterior side of the tibia, using this area as the region of interest (ROI) for quantitative analysis of relative bone volume (BV/TV).

\section{Flow Cytometry}

Take the tumor-draining lymph node and grind with the rubber end of the syringe to obtain a single-cell suspension. Tumor tissue was obtained, the tumor was minced with a scalpel, and treated with $1 \mathrm{mg} / \mathrm{mL}$ collagenase I (Gibco, United States) for $1 \mathrm{~h}$. The cells were filtered through a nylon mesh filter and washed with PBS. The single-cell suspension was blocked with anti-CD16/32 (Invitrogen) on ice for $20 \mathrm{~min}$ to reduce non-specific binding to the immunoglobulin Fc receptor. The cells were further stained with antibodies conjugated to the following fluorescent dyes: CD45, CD3e, CD4, CD8 (all from Invitrogen). Flow cytometry was performed on LSRII (BD Biosciences), and data analysis was performed using FlowJo software (TreeStar).

\section{Pathological Analysis}

On the 16th day after orthotopic injection of $\mathrm{mPEG}_{45}-\mathrm{PLV}_{19}$ gel, all mice were euthanized. The main organs and tumors were collected, paraformaldehyde $(4.0 \%)$ was used to fix the main organs and tumor tissues, hematoxylin and eosin (H\&E) were used to stain the tumors and organ sections, and the microscope (Nikon Eclipse Ti, Ardmore, PA) observes the changes in histomorphology.

\section{RESULTS AND DISCUSSION}

\section{Degradation, Drug Release, and Cytotoxicity of the Gel in vitro}

We analyzed the in vitro degradation properties of the gel. The result is shown in Figure 1A. We found that all three groups of gels exhibited similar degradation rates in the buffer solution of simulated body fluids. The degradation process was stable, and there was no rapid weight loss at the initial stage of degradation. And the degradation rate reached $50 \%$ in about 27 days.

The results of drug release in vitro are shown in Figures 1B,C. The drug-loaded in gel achieves a sustained-release effect. None of the three groups of drug-loaded gels showed the rapid release of early drugs. The gel loaded with OXA and/or ALN slowly released about $40 \%$ of the drug within 15 days.

We verified the effect of gel on K7M2 cell activity through MTT assay. The result is shown in Figure 1D. We measured the cell activity at 0,24 , and $48 \mathrm{~h}$, and the results showed that the existence of gel does not affect the activity of tumor cells.

The above experiments show that $\mathrm{mPEG}_{45}-\mathrm{PLV}_{19}$ temperature-sensitive gel is an ideal drug-loaded gel. It has an appropriate degradation rate and drug release rate and can achieve sustained release of drugs in situ, which is conducive to maintaining local drug concentration to achieve the ideal therapeutic effect.

\section{Evaluation of Thermosensitive Hydrogel Synergistic Delivery of Oxaliplatin and Alendronate Anti-tumor Effects in vivo}

After the experiment, the mice were euthanized. The osteosarcoma tissue in situ was excised and photographed. As shown in Figure 2A, the tumor volume progression of each treatment group was slower than that of the blank group. Among them, the OXA + ALN group showed an unfortunate tumor-suppressive effect than other three treatment groups, 
A

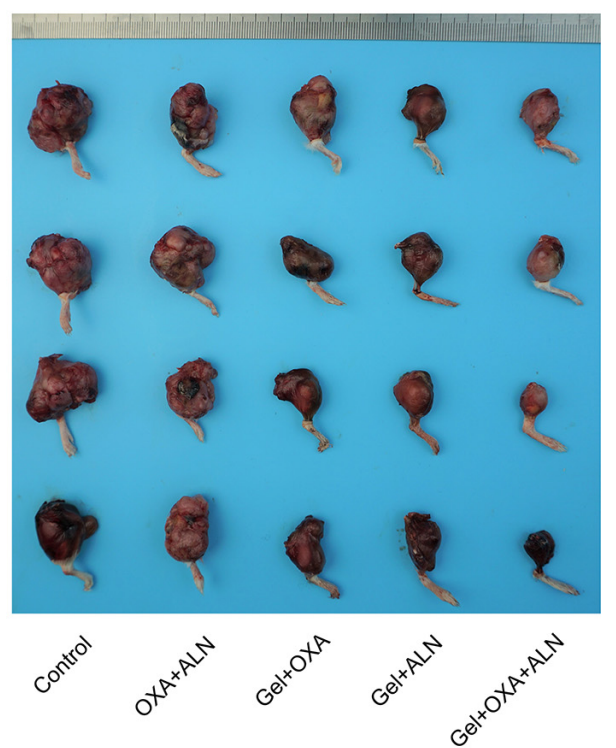

C

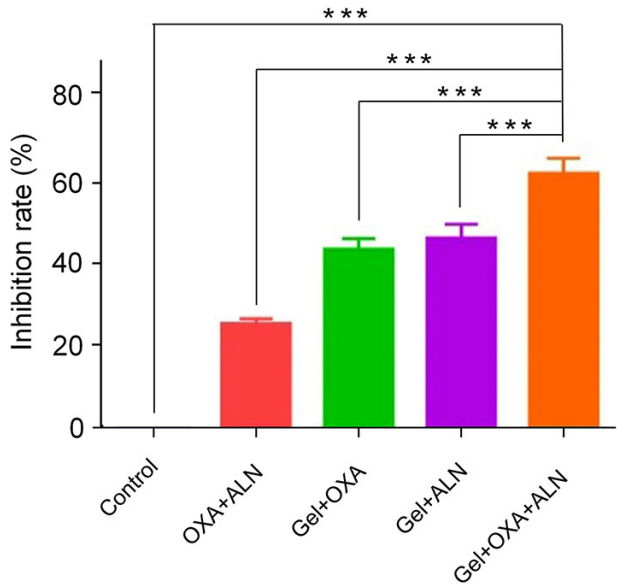

B

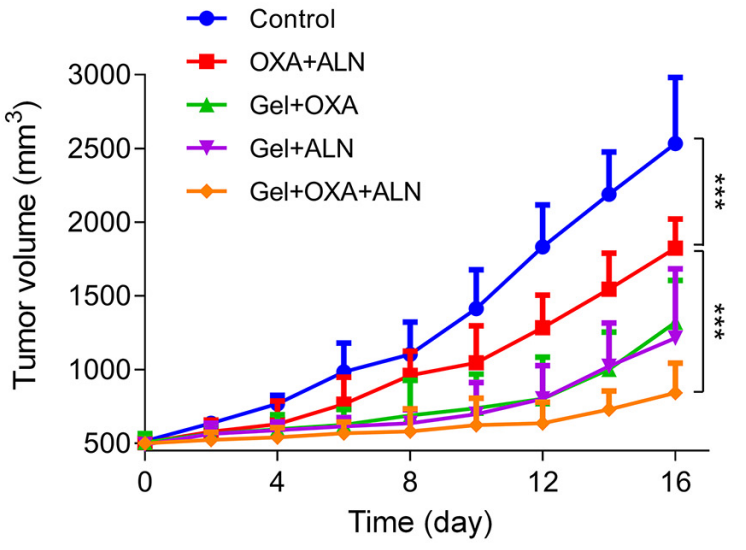

D

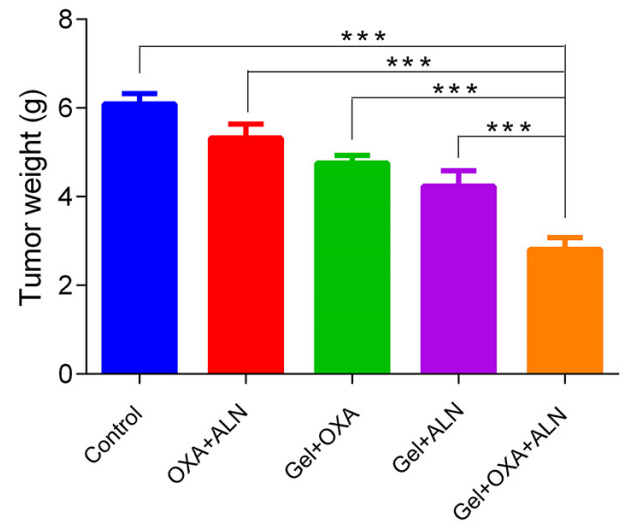

FIGURE 2 | (A) Tumor pictures after treatment in each group. The inhibition curve (B), tumor inhibition rate (C), and the weight of osteosarcoma after treatment (D) of each group, the data is expressed as mean $\pm \mathrm{SD}\left(n=4\right.$; $\left.{ }^{\star \star \star} P<0.001\right)$.

which indicates that it is necessary to use the gel to achieve local sustained-release drugs. A single local injection of free drugs will not achieve satisfactory therapeutic effects due to faster metabolism. In the Gel + OXA + ALN group, The growth rate of tumor volume achieved the most obvious slowed down, which suggested that the gel targeted delivery of OXA and ALN had a significant effect on inhibiting the formation of OS.

The tumor volume was measured as shown in Figure 2B. On the first day, the initial volume of each group was $514.4 \pm 48.1 \mathrm{~mm}^{3}$ (Control group), $504.2 \pm 56.9 \mathrm{~mm}^{3}$ (OXA + ALN group), $507.1 \pm 59.6 \mathrm{~mm}^{3}$ (Gel + OXA group), $491.6 \pm 45.2 \mathrm{~mm}^{3}$ (Gel + ALN group), and $499.6 \pm 33.5 \mathrm{~mm}^{3}$ (Gel + OXA + ALN group), the tumor volume baseline was the same. At half of the experiment, on the 8th day after treatment, the tumor volume of each group was $1102.5 \pm 220.3 \mathrm{~mm}^{3}$ (Control group),
$962.1 \pm 162.7 \mathrm{~mm}^{3}$ (OXA + ALN group), $689.2 \pm 238.2 \mathrm{~mm}^{3}$ (Gel + OXA group), $635.1 \pm 93.1 \mathrm{~mm}^{3}$ (Gel + ALN group) and $580.2 \pm 153.9 \mathrm{~mm}^{3}$ (Gel + OXA + ALN group). In 8 days, the tumor volume of Control group mice increased rapidly, and the volume increase was as high as $3139.3 \mathrm{~mm}^{3}$. After the gel was loaded with OXA or ALN alone, the tumor was effectively controlled. In the Gel + OXA + ALN group, the tumor-suppressing effect was the best. At the end of the experiment, on the 16th day after treatment, the tumor volume in the Gel + OXA + ALN group was $841.2 \pm 202.9 \mathrm{~mm}^{3}$, which was significantly smaller than that in the Gel + ALN group $1214.7 \pm 468.5 \mathrm{~mm}^{3}$, in the $\mathrm{Gel}+$ OXA group $1320.6 \pm 285.1 \mathrm{~mm}^{3}$, and OXA + ALN group $1824.8 \pm 197.5 \mathrm{~mm}^{3}$. The tumor volume of the untreated control group was $2533.3 \pm 448.1 \mathrm{~mm}^{3}$.

As shown in Figure 2C, there was a significant difference in the tumor inhibition rate among each group. The tumor inhibition 
A

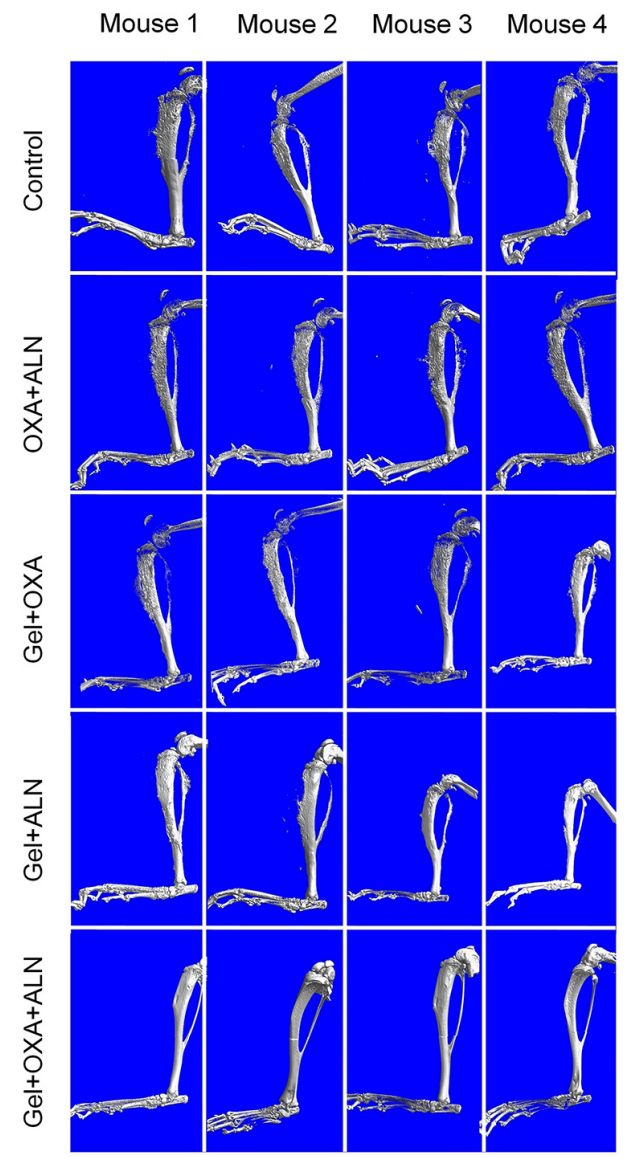

B

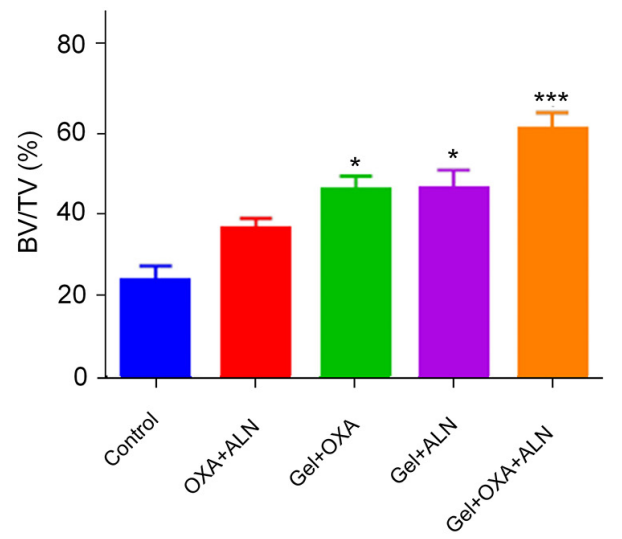

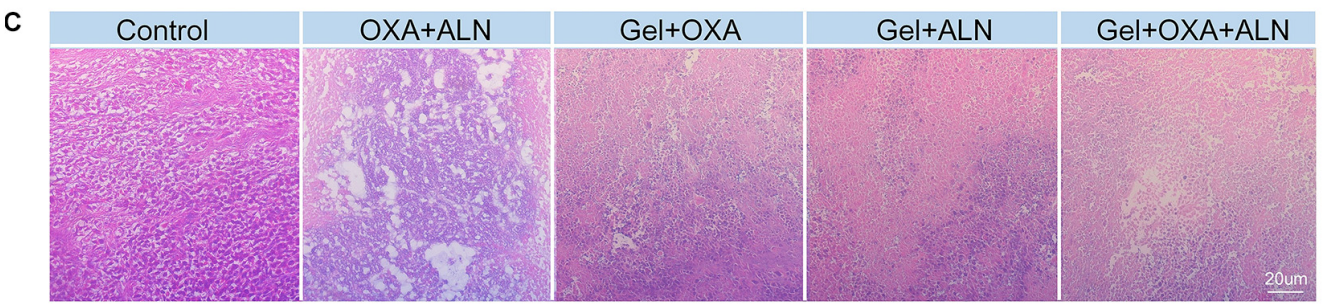

FIGURE 3 | (A) Micro-CT images of orthotopic osteosarcoma after treatment in each group. (B) Relative bone volume of tibia of each treatment group, the data is expressed as mean $\pm \mathrm{SD}\left(n=4\right.$. Compared with Control group, $\left.{ }^{\star} P<0.05,{ }^{\star \star \star} P<0.001\right)$. (C) Orthotopic tumor histopathology images after treatment in each group.

rate of the OXA + ALN group was only 25.2\%, and the tumor inhibition rates of the gel loaded with OXA and ALN alone were $42.0 \%$ and $43.8 \%$, respectively. The Gel + MTX + ALN group had a tumor suppression rate of $60.4 \%$, achieving the most significant tumor inhibition effect. The results indicated that the sustained release of gel and the combined use of synergistic drugs could maximize the therapeutic impact of in situ tumors.

The dissected tumor is weighed. As shown in Figure 2D, compared with other groups, the average weight of hind limbs with tumors in the Gel + OXA + ALN group was the smallest $(2.81 \pm 0.26 \mathrm{~g})$. OXA + ALN group $(5.32 \pm 0.31 \mathrm{~g})$ and control group $(6.09 \pm 0.23 \mathrm{~g})$ tumor-containing hindlimb weights were close, and the results were consistent with tumor volume changes.

\section{Evaluation of Anti-bone Destruction \\ Effects}

To further confirm the anti-tumor efficacy of Gel + OXA + ALN, the degree of bone destruction caused by local OS invasion was evaluated by micro-CT. As shown in Figure $\mathbf{3 A}$, severe pathological bone destruction induced by OS progression was found in the proximal tibia of the control group mice without any treatment. The OXA + ALN group also had serious pathological bone destruction. In the Gel + OXA group, Gel + ALN group, and $\mathrm{Gel}+\mathrm{OXA}+\mathrm{ALN}$ 
A

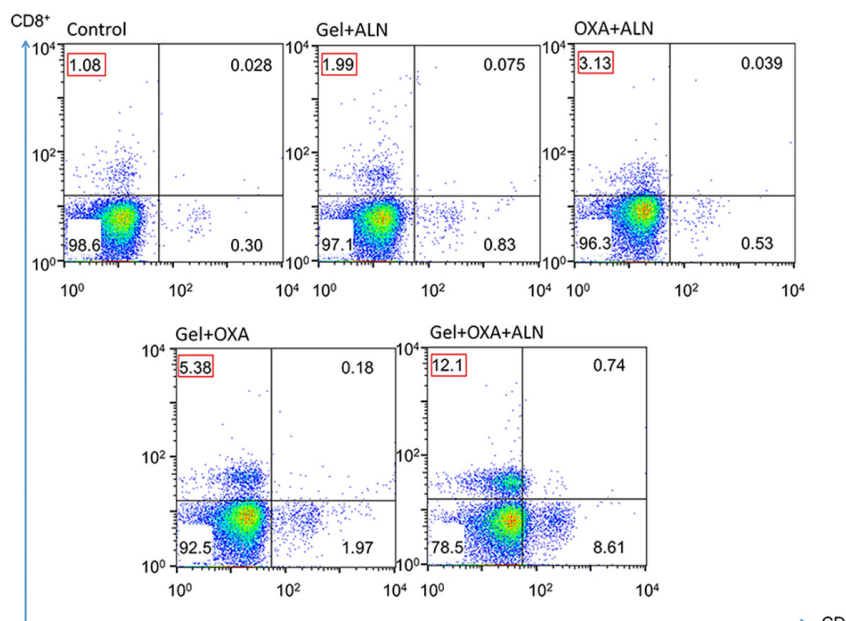

C

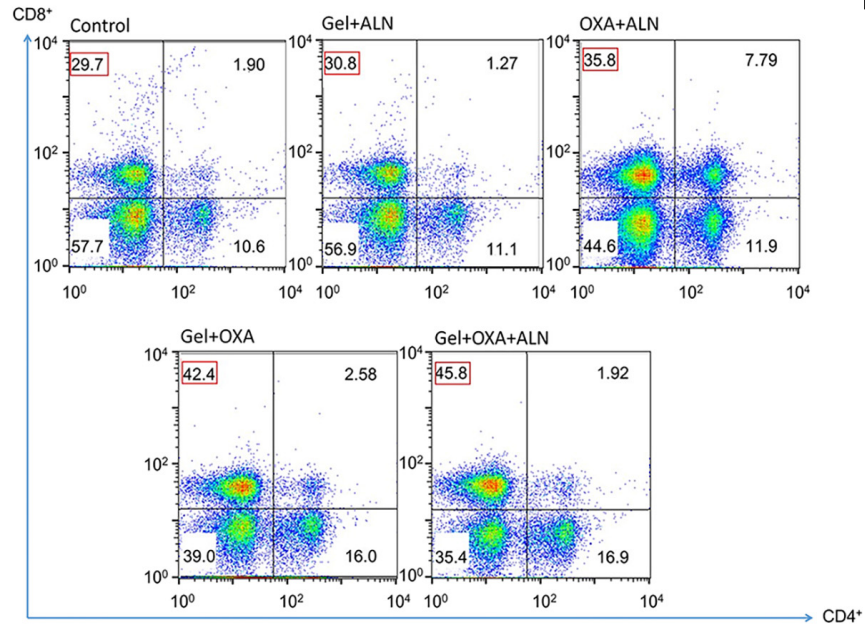

B

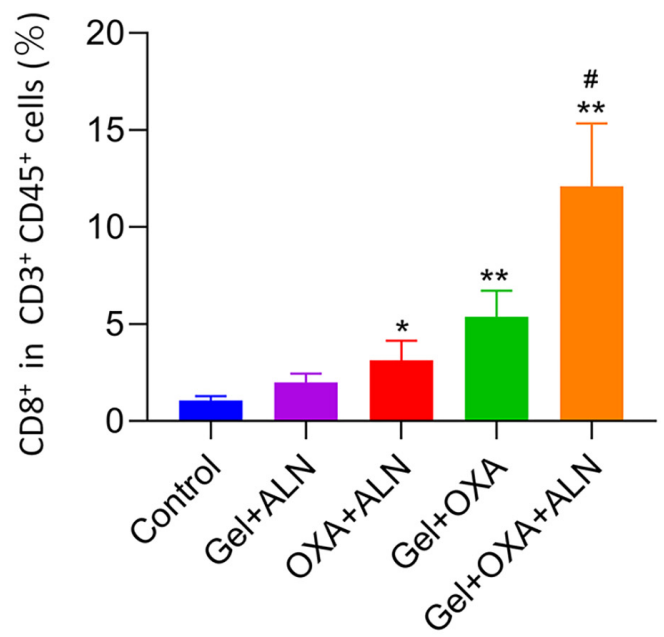

D

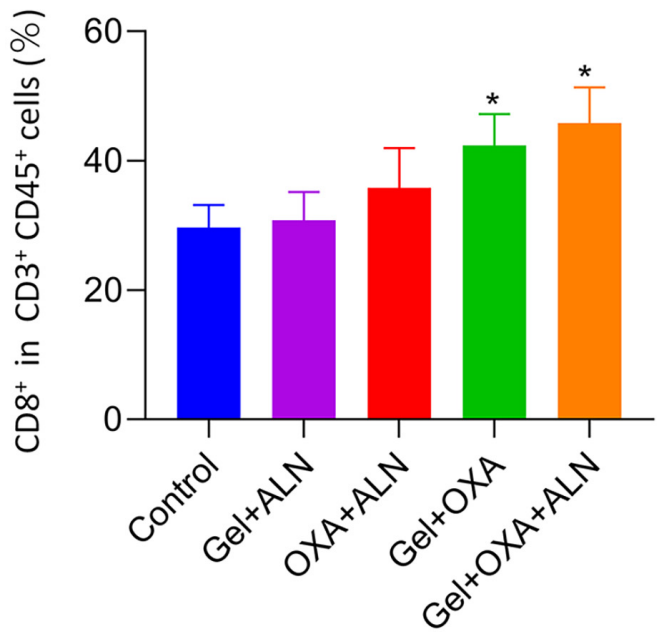

FIGURE 4 | In situ OS were collected from mice 16 days after treatment. Representative flow cytometry analysis images (A) and the relative quantification gating on $\mathrm{CD}^{+}$cells in $\mathrm{CD}^{+} \mathrm{CD}^{+} 5^{+}$cells (B). Data are presented as mean $\pm \mathrm{SD}(n=3)$. Lymph nodes adjacent to the tumor were collected from mice 16 days after treatment. Representative flow cytometry analysis images (C) and the relative quantification gating on $\mathrm{CD}^{+}$cells in $\mathrm{CD} 3^{+} \mathrm{CD} 45^{+}$cells (D). Data are presented as mean $\pm \mathrm{SD}(n=3)$. Compared with Control group, ${ }^{\star} P<0.05,{ }^{\star \star} P<0.01$. Compared with Gel + ALN group, ${ }^{\#} P<0.05$.

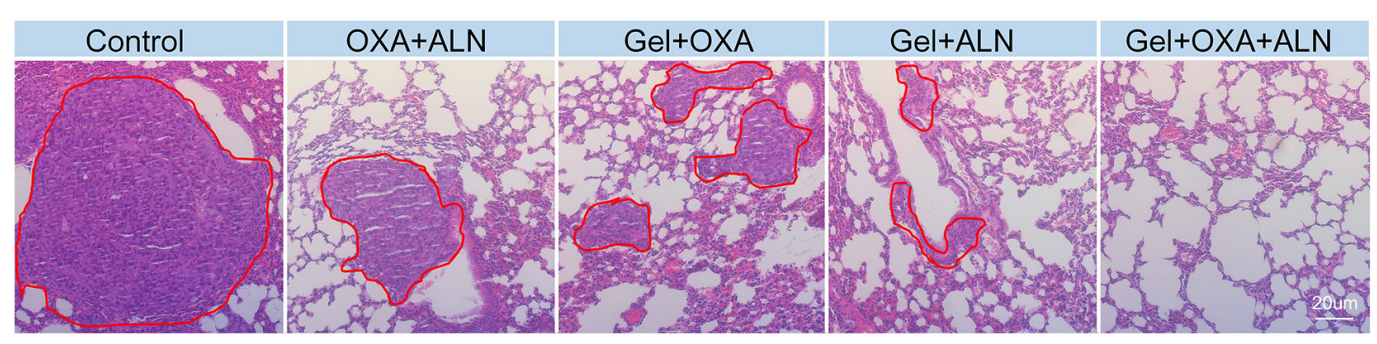

FIGURE $\mathbf{5}$ | H\&E images of lungs tissue in different treatment groups (Lungs metastases are marked in the red circle). 


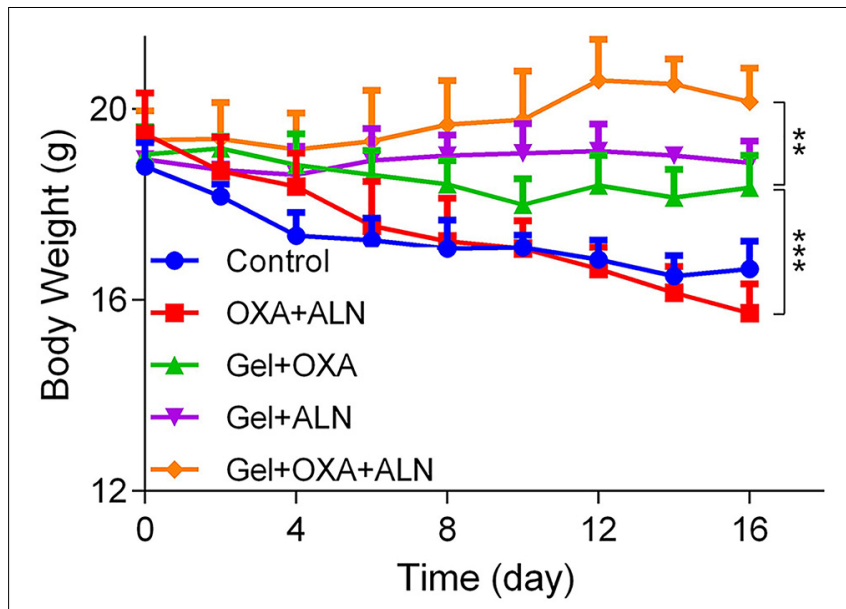

FIGURE 6 | Body weight curve of each treatment group, the data is expressed as mean $\pm \operatorname{SD}\left(n=4,{ }^{* \star} P<0.01,{ }^{* \star *} P<0.001\right)$.

group, the proximal tibia bone destruction of the lower limbs of the mice was weakened, and the degree of bone destruction in the Gel + OXA + ALN group was the most limited. The results of micro-CT indicate that the Gel + OXA + ALN group has the best protective effect on OS bone destruction. Similarly, as shown in Figure 3B, the relative bone volume (BV/TV) values of each group were compared: Control group $(21.86 \% \pm 2.87 \%)$, OXA + ALN group (34.22\% $\pm 1.92 \%)$, Gel + OXA group $(43.09 \% \pm 2.38 \%)$, Gel + ALN group $(43.51 \% \pm 3.33 \%)$ and $\mathrm{Gel}+\mathrm{OXA}+\mathrm{ALN}$ group $(56.61 \% \pm 3.17 \%)$. We can also come to a similar conclusion that the degree of bone destruction in Gel + OXA + ALN group was the most limited, indicating the $\mathrm{Gel}+\mathrm{OXA}+\mathrm{ALN}$ provided the most potent protective effect on bone destruction.

In order to explain the reasons for the protection of bone tissue by this system, we further explored the inhibitory effect of the system on osteosarcoma tissue. OS in situ of mice was fixed with formaldehyde, then decalcified, embedded, sectioned, and finally stained by H\&E. After that, the histopathological analysis was performed. The results are shown in Figure 3C. No necrosis-related symptoms were seen in the control group. In situ OS tissues of mice in the OXA + ALN group, Gel + OXA group, Gel + ALN group all had different degrees of necrosis. In the Gel + OXA + ALN group, severe cell atrophy, reduced density, nucleocytoplasmic concentration, and even tumor cell disappearance were observed. A large area of tumor cell apoptosis and necrosis were also showed. Therefore, the histopathological analysis further confirmed the anti-tumor efficacy and the potential of Gel + OXA + ALN for in situ anti-tumor treatment.

The results of tumor volume, weight, H\&E staining, and pathological bone destruction related to the progression of the proximal tibia of the lower extremity and OS emphatically demonstrated the anti-tumor efficacy of Gel + OXA + ALN and the ability of inhibiting the bone destruction caused by OS.

\section{Evaluation of Anti-tumor Immune Effects}

Tumor cells and lymphocytes of mice were collected for flow cytometry experiments. The anti-tumor immune effect caused by OXA was clarified by analyzing lymphocytes. After the treatment, the tumor tissues were isolated under sterile conditions and digested with collagenase to prepare a single tumor cell suspension. Flow cytometry was performed by antibody labeling, and $\mathrm{CD}^{+}$and $\mathrm{CD}^{+} 5^{+}$, double-positive cells were analyzed. Representative flow cytometry analysis images were shown in Figure 4A, the $\mathrm{CD} 8^{+} \mathrm{T}$ cell infiltration rate of the tumor in the control group was $1.08 \%$. The rate of $\mathrm{CD}^{+} \mathrm{T}$ cell infiltration induced by Gel + ALN injection near the tumor was 1.99\%, no significant difference was observed with the control group. However, the injection rate of free OXA + ALN beside the tumor-induced the infiltration rate of $\mathrm{CD}^{+}{ }^{+} \mathrm{T}$ cells to be $3.31 \%$, confirming that OXA could induce ICD and increase the number of $\mathrm{CD}^{+} \mathrm{T}$ lymphocytes in the tumor. However, since a single local injection of free drugs will be rapid metabolized, free OXA + ALN group has not achieved satisfactory therapeutic effects. Gel + OXA induced $\mathrm{CD}^{+} \mathrm{T}$ cell infiltration rate to $5.38 \%$, indicating that the gel can slowly release OXA, causing the sustained anti-tumor immune effect. The Gel + OXA + ALN group induced a $\mathrm{CD}^{+} \mathrm{T}$ cell infiltration rate of $12.1 \%$, which was higher than any other treatment group. The reason may be that ALN has an inhibitory effect on the growth of osteosarcoma, which can indirectly increase the proportion of lymphocytes in the tumor and reduce the tumor's resistance to immune cells. So that OXA and ALN have a synergistic therapeutic effect. We performed the above tests on three mice in each group, and the results were shown in Figure 4B. We performed a statistical analysis of the results and verified the above analysis results again.

In order to further verify the immune effect of the treatment, we collected tumor-draining lymph node lymphocytes for flow cytometry analysis. As shown in Figure $4 \mathrm{C}, \mathrm{CD}^{+}$and $\mathrm{CD} 45^{+}$double-positive cells were used as analysis objects. The $\mathrm{Gel}+\mathrm{OXA}+\mathrm{ALN}$ treatment group had the highest $\mathrm{CD}^{+}{ }^{+} \mathrm{T}$ cell infiltration rate $(45.8 \%)$, higher than the Gel + OXA group (42.4\%), free OXA + ALN group (35.8\%), and Gel + ALN group (30.8\%) and control group (29.7\%). The results are consistent with the infiltration of CD8 ${ }^{+} \mathrm{T}$ cells in tumor cells, which proves once again that OXA induces ICD in tumor cells, stimulates the body's anti-tumor immune response and achieves the goal of inhibiting OS progression. We performed the above tests on three mice in each group, and the results were shown in Figure 4D. We performed a statistical analysis on the results and verified the above analysis results again.

\section{Evaluation of the Inhibiting Lung Metastasis Effects}

OS is often accompanied by lung metastasis, which significantly affects the prognosis of patients in clinic. The lung tissues were fixed, sectioned, and $\mathrm{H} \& \mathrm{E}$ stained for pathological analysis. As shown in Figure 5, the number and area of tumors in the lung sections of the Gel + OXA + ALN group were the smallest, showing that Gel + OXA + ALN had a more profound inhibitory effect on OS lung metastasis than other treatment groups. 


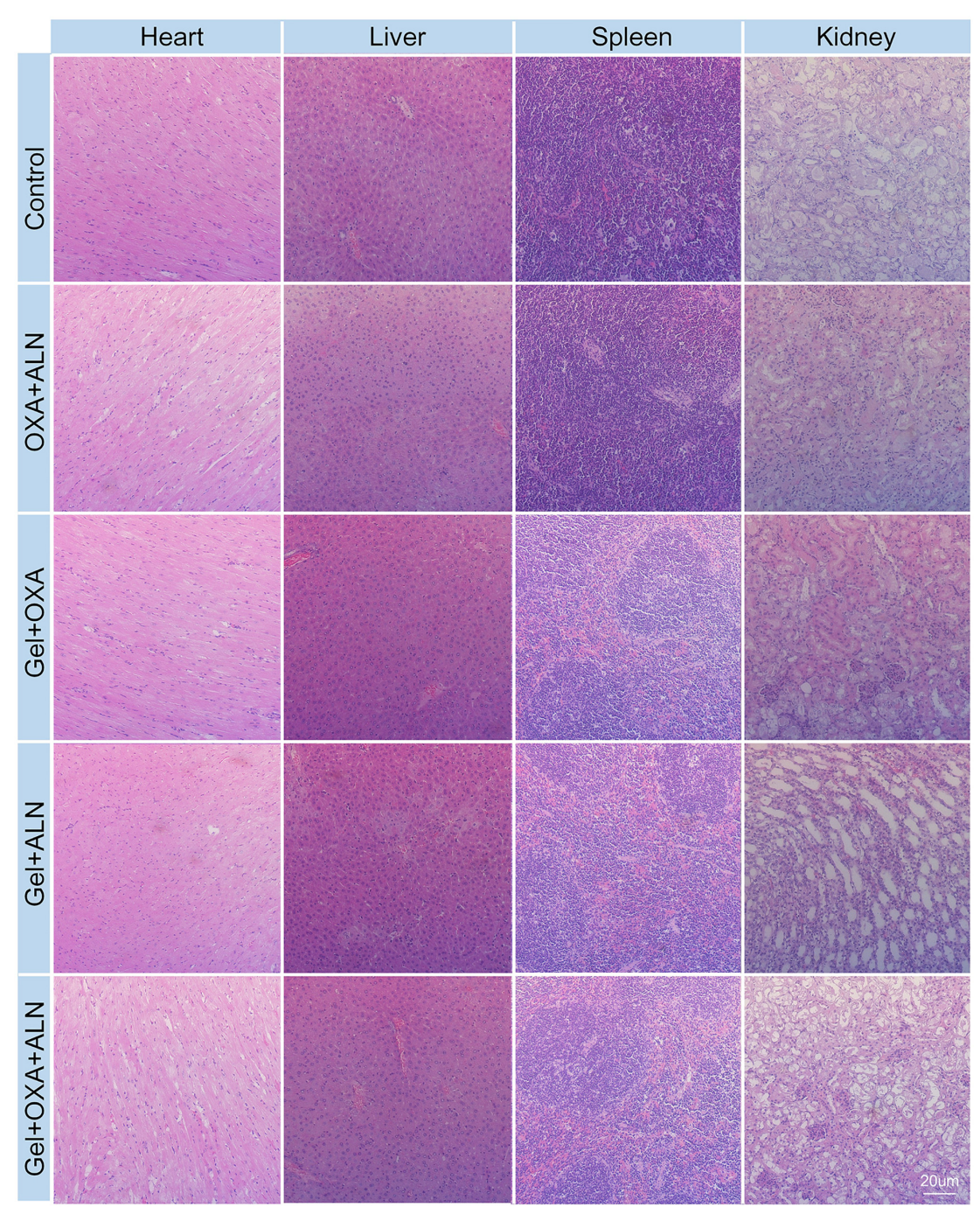

FIGURE 7 | Histopathological analysis of organs in each treatment group.

\section{Evaluation of the Safety in vivo}

Safety is a critical evaluation index for drug delivery systems. We verified the safety of Gel + OXA + ALN through weight monitoring and histopathological examination of several susceptible organs. As shown in Figure 6, the bodyweight of the mice in the control group showed a significant decrease because of no treatment. The malignant transformation of the tumor is very serious, and the tumor seriously damages the health of the mice. Due to the toxicity of free drugs, the OXA + ALN group had the most obvious weight-loss trend. The Gel + OXA and Gel + ALN groups showed different degrees of weight loss. The Gel + OXA + ALN group showed the best stable body weight and a slight weight increase. The H\&E staining results of the main organs (heart, liver, spleen, kidney) could also indicate the toxicity of the longterm application of various therapeutic drugs to the body. As shown in Figure 7, we found that there were no noticeable morphological changes in the heart, liver, spleen and kidney of the tumor-bearing mice in the Gel + OXA + ALN group compared with the control group. The above results conclusively prove the low toxicity of Gel + OXA + ALN to all major organs in the whole body, indicating that it has great potential for clinical application.

\section{CONCLUSION}

In this study, a system of synergistic in situ treatment of osteosarcoma with $\mathrm{mPEG}_{45}-\mathrm{PLV}_{19}$ thermosensitive hydrogel loaded with OXA and ALN was established. OXA can improve the immunogenicity of tumor and promote the accumulation of cytotoxic T cells, ALN can synergistically diminish tumors and has a bone-targeting effect to relieve bone destruction. The synergistic effect of them increases the therapeutic effect of this system on OS. The Gel + OXA + ALN group showed the highest anti-tumor activity and the most reliable safety in vivo in the 
mouse in situ OS model. The effective combination of $\mathrm{mPEG}_{45}-$ $\mathrm{PLV}_{19}$ thermo-sensitive hydrogel with OXA and ALN provides broad prospects for in situ anti-osteosarcoma treatment.

\section{DATA AVAILABILITY STATEMENT}

All datasets generated for this study are included in the article/supplementary material.

\section{ETHICS STATEMENT}

The animal study was reviewed and approved by the Animal Care and Use Committee at the Jilin University.

\section{REFERENCES}

Blanco, E., Kessinger, C. W., Sumer, B. D., and Gao, J. (2009). Multifunctional micellar nanomedicine for cancer therapy. Medicine 234, 123-131. doi: 10.3181/ 0808-MR-250

Cheng, C., Ding, Q., Zhang, Z., Wang, S., Zhong, B., Huang, X., et al. (2020). PTBP1 modulates osteosarcoma chemoresistance to cisplatin by regulating the expression of the copper transporter SLC31A1. J. Cell Mol. Med. 24, 5274-5289. doi: $10.1111 /$ jcmm. 15183

Feather, C. E., Lees, J. G., Makker, P. G. S., Goldstein, D., Kwok, J. B., MoalemTaylor, G., et al. (2018). Oxaliplatin induces muscle loss and muscle-specific molecular changes in Mice. Muscle Nerve 57, 650-658. doi: 10.1002/mus.25966

Ghiringhelli, F., Apetoh, L., Tesniere, A., Aymeric, L., Ma, Y., Ortiz, C., et al. (2009). Activation of the NLRP3 inflammasome in dendritic cells induces IL1 beta-dependent adaptive immunity against tumors. Nat. Med. 15, 1170-1178. doi: $10.1038 / \mathrm{nm} .2028$

Gomez-Brouchet, A., Illac, C., Gilhodes, J., Bouvier, C., Aubert, S., Guinebretiere, J. M., et al. (2017). CD163-positive tumor-associated macrophages and CD8positive cytotoxic lymphocytes are powerful diagnostic markers for the therapeutic stratification of osteosarcoma patients: an immunohistochemical analysis of the biopsies fromthe French OS 2006 trial. Clin. Cancer Res. 24:69. doi: 10.1080/2162402X.2017.1331193

Harrison, D. J., Geller, D. S., Gill, J. D., Lewis, V. O., and Gorlick, R. (2018). Current and future therapeutic approaches for osteosarcoma. Expert. Rev. Anticancer Ther. 18, 39-50. doi: 10.1080/14737140.2018.1413939

Hato, S. V., Khong, A., De Vries, I. J. M., and Lesterhuis, W. J. (2014). Molecular pathways: the immunogenic effects of platinum-based chemotherapeutics. Clin. Cancer Res. 20, 2831-2837. doi: 10.1158/1078-0432.CCR-13-3141

Huang, H., Qi, X., Chen, Y., and Wu, Z. (2019). Thermo-sensitive hydrogels for delivering biotherapeutic molecules: a review. Saudi Pharm. J. 27, 990-999. doi: 10.1016/j.jsps.2019.08.001

Huang, X., Zhao, J., Bai, J., Shen, H., Zhang, B., Deng, L., et al. (2019). Risk and clinicopathological features of osteosarcoma metastasis to the lung: a population-based study. J. Bone Oncol. 16:100230. doi: 10.1016/j.jbo.2019. 100230

Jiang, Z., Feng, X., Xu, W., Zhuang, X., Ding, J., and Chen, X. (2020). Calcium phosphate-cured nanocluster of poly(l-glutamic acid)-cisplatin and arsenic trioxide for synergistic chemotherapy of peritoneal metastasis of ovarian cancer. Acta Polym. Sin. 51, 901-910. doi: 10.11777/j.issn1000-3304.2020.20053

Kansara, M., Teng, M. W., Smyth, M. J., and Thomas, D. M. (2014). Translational biology of osteosarcoma. Nat. Rev. Cancer 14, 722-735. doi: 10.1038/nrc3838

Koirala, P., Roth, M. E., Gill, J., Piperdi, S., Chinai, J. M., Geller, D. S., et al. (2016). Immune infiltration and PD-L1 expression in the tumor microenvironment are prognostic in osteosarcoma. Sci. Rep. 6:30093. doi: 10.1038/srep30093

Li, K., Li, D., Zhao, L., Chang, Y., Zhang, Y., Cui, Y., et al. (2020). Calcium-mineralized polypeptide nanoparticle for intracellular drug delivery in osteosarcoma chemotherapy. Bioact. Mater. 5, 721-731. doi: 10.1016/j. bioactmat.2020.04.010

\section{AUTHOR CONTRIBUTIONS}

YS, CL, YZ, and DZ proposed and designed the experiments. YS, $\mathrm{KL}$, and $\mathrm{DZ}$ carried out the experiments with the help of $\mathrm{CL}$ and YZ. YS, KL, and DZ drafted the manuscript and interpreted the data. CL and YZ revised the manuscript. All authors contributed to the article and approved the submitted version.

\section{FUNDING}

This work was financially supported by the Specialized Direct Subordinated Program from Finance Department of Jilin Province (Grant No. 3D518V313429).

Li, S. Q., Dong, S. J., Xu, W. G., Tu, S. C., Yan, L. S., Zhao, C. W., et al. (2018). Antibacterial hydrogels. Adv. Sci. 5:1700527. doi: 10.1002/advs.201700527

Liu, H., Cheng, Y., Chen, J., Chang, F., Wang, J., Ding, J., et al. (2018). Component effect of stem cell-loaded thermosensitive polypeptide hydrogels on cartilage repair. Acta Biomater. 73, 103-111. doi: 10.1016/j.actbio.2018.04.035

Martins, I., Wang, Y., Michaud, M., Ma, Y., Sukkurwala, A. Q., Shen, S., et al. (2014). Molecular mechanisms of ATP secretion during immunogenic cell death. Cell Death. Differ 21, 79-91. doi: 10.1038/cdd.2013.75

Menger, L., Vacchelli, E., Adjemian, S., Martins, I., Ma, Y., Shen, S., et al. (2012). Cardiac glycosides exert anticancer effects by inducing immunogenic cell death. Sci. Transl. Med. 4:143ra99. doi: 10.1126/scitranslmed.3003807

Mouw, K. W., Goldberg, M. S., Konstantinopoulos, P. A., and D'Andrea, A. D. (2017). DNA damage and repair biomarkers of immunotherapy response. Cancer Discov. 7, 675-693. doi: 10.1158/2159-8290.Cd-17-0226

Ning, C., Guo, Y., Yan, L., Thawani, J. P., Zhang, W., Fu, C., et al. (2019). On-demand prolongation of peripheral nerve blockade through bupivacaineloaded hydrogels with suitable residence periods. ACS Biomater. Sci. Eng. 5, 696-709. doi: 10.1021/acsbiomaterials.8b01107

Qiu, H., Guo, H., Li, D., Hou, Y., Kuang, T., and Ding, J. (2020). Intravesical hydrogels as drug reservoirs. Trends Biotechnol. 38, 579-583. doi: 10.1016/j. tibtech.2019.12.012

Shan, H., Li, K., Zhao, D., Chi, C., Tan, Q., Wang, X., et al. (2020). Locally controlled release of methotrexate and alendronate by thermo-sensitive hydrogels for synergistic inhibition of osteosarcoma progression. Front. Pharmacol. 11:573. doi: 10.3389/fphar.2020.00573

Sukkurwala, A. Q., Martins, I., Wang, Y., Schlemmer, F., Ruckenstuhl, C., Durchschlag, M., et al. (2014). Immunogenic calreticulin exposure occurs through a phylogenetically conserved stress pathway involving the chemokine CXCL8. Cell Death. Differ 21, 59-68. doi: 10.1038/cdd.2013.73

Tinkle, C. L., Lu, J., Han, Y., Li, Y., McCarville, B. M., Neel, M. D., et al. (2019). Curative-intent radiotherapy for pediatric osteosarcoma: the St. Jude experience. Pediatr. Blood Cancer. 66:e27763. doi: 10.1002/pbc.27763

Uihlein, A. V., and Leder, B. Z. (2012). Anabolic therapies for osteoporosis. Endocrinol. Metab. Clin. North Am. 41, 507-525. doi: 10.1016/j.ecl.2012. 05.002

van Erp, A. E. M., Versleijen-Jonkers, Y. M. H., Hillebrandt-Roeffen, M. H. S., van Houdt, L., Gorris, M. A. J., van Dam, L. S., et al. (2017). Expression and clinical association of programmed cell death-1, programmed death-ligand-1 and CD8(+) lymphocytes in primary sarcomas is subtype dependent. Oncotarget 8 , 71371-71384. doi: 10.18632/oncotarget.19071

Wang, C., Feng, N., Chang, F., Wang, J., Yuan, B., Cheng, Y., et al. (2019). Injectable cholesterol-enhanced stereocomplex polylactide thermogel loading chondrocytes for optimized cartilage regeneration. Adv. Healthc. Mater. 8:1900312. doi: 10.1002/adhm.201900312

Wang, W., Ding, H., Sun, Z., Jin, C., Zhu, Y., and Wang, X. (2020). A populationbased propensity-matched study of regional dissections in patients with metastatic osteosarcoma. J. Orthop. Surg. Res. 15:107. doi: 10.1186/s13018-020$01592-y$ 
Wang, Y., Jiang, Z., Xu, W., Yang, Y., Zhuang, X., Ding, J., et al. (2019). Chiral polypeptide thermogels induce controlled inflammatory response as potential immunoadjuvants. ACS Appl. Mater. Interfaces 11, 8725-8730. doi: 10.1021/ acsami.9b01872

Wang, Y. K., Qin, S. Q., Ma, T., Song, W., Jiang, R. Q., Guo, J. B., et al. (2017). Effects of teriparatide versus alendronate for treatment of postmenopausal osteoporosis: a meta-analysis of randomized controlled trials. Medicine 96:e6970. doi: 10.1097/md.0000000000006970

Wang, Y. K., Zhang, Y. M., Qin, S. Q., Wang, X., Ma, T., Guo, J. B., et al. (2018). Effects of alendronate for treatment of glucocorticoid-induced osteoporosis: a meta-analysis of randomized controlled trials. Medicine 97:e12691. doi: 10. 1097/md.0000000000012691

Wei, L., Chen, J., Zhao, S., Ding, J., and Chen, X. (2017). Thermo-sensitive polypeptide hydrogel for locally sequential delivery of two-pronged antitumor drugs. Acta Biomater. 58, 44-53. doi: 10.1016/j.actbio.2017.05.053

Zhang, B., Zhang, Y., Li, R., Li, J., Lu, X., and Zhang, Y. (2020). The efficacy and safety comparison of first-line chemotherapeutic agents (highdose methotrexate, doxorubicin, cisplatin, and ifosfamide) for osteosarcoma: a network meta-analysis. J. Orthop. Surg. Res. 15:51. doi: 10.1186/s13018-0201576-0
Zhang, W., Cong, N., Xu, W., Hu, H., Li, M., Zhao, G., et al. (2018). Precisionguided long-acting analgesia by hydrogel-immobilized bupivacaine-loaded microsphere. Theranostics 8, 3331-3347. doi: 10.7150/thno.25276

Zhang, Y., Yu, J., Ren, K., Zuo, J., Ding, J., and Chen, X. (2019). Thermosensitive hydrogels as scaffolds for cartilage tissue engineering. Biomacromolecules 20, 1478-1492. doi: 10.1021/acs.biomac.9b00043

Zheng, P., Liu, Y., Chen, J., Xu, W., Li, G., and Ding, J. (2020). Targeted pHresponsive polyion complex micelle for controlled intracellular drug delivery. Chin. Chem. Lett. 31, 1178-1182. doi: 10.1016/j.cclet.2019.12.001

Conflict of Interest: The authors declare that the research was conducted in the absence of any commercial or financial relationships that could be construed as a potential conflict of interest.

Copyright (C) 2020 Sun, Li, Li, Zhang and Zhao. This is an open-access article distributed under the terms of the Creative Commons Attribution License (CC BY). The use, distribution or reproduction in other forums is permitted, provided the original author(s) and the copyright owner(s) are credited and that the original publication in this journal is cited, in accordance with accepted academic practice. No use, distribution or reproduction is permitted which does not comply with these terms. 